Führung in Familienunternehmen: BETRIEBSWIRTSCHAFT

\title{
Besonderheiten der Entscheidungsfindung und Verhaltenssteuerung und deren Auswirkung auf den Unternehmenserfolg
}
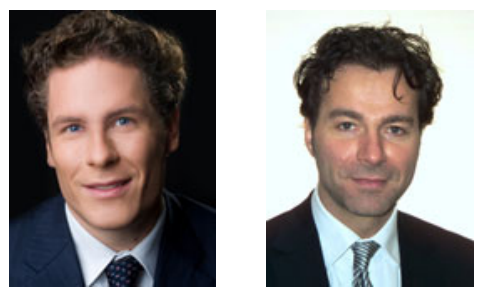

Arthur Posch • Gerhard Speckbacher

Zusammenfassung: Der vorliegende Beitrag untersucht anhand einer empirischen Studie, wie sich die Dominanz einer Gründerfamilie als Eigentümer und in der Geschäftsführung auf die Nutzung von Daten und Kennzahlen zur Rationalitätssicherung bei der Entscheidungsfindung auf Geschäftsführungsebene sowie auf die Verhaltenssteuerung von Mitarbeiter/inne/n auswirkt. Zudem wird untersucht, ob Familienunternehmen, deren Geschäftsführung Entscheidungen weniger intuitiv und stärker kennzahlenorientiert trifft, tendenziell erfolgreicher sind und welcher Zusammenhang zwischen der Verwendung der untersuchten Verhaltenssteuerungsinstrumente und dem Erfolg von Familienunternehmen besteht. Unsere Untersuchung liefert empirische Evidenz, dass Familienunternehmen tatsächlich Instrumente zur Entscheidungsunterstützung weniger nutzen als NichtFamilienunternehmen und dass auch signifikante Unterschiede bei der Art der Verhaltenssteuerung von Mitarbeiter/inne/n existieren. Allerdings relativiert die vorliegende Studie die in der Literatur zu Familienunternehmen häufig zu findende Argumentation, wonach Familienunternehmen erfolgreicher sein könnten, wenn sie sich ähnlich wie Nichtfamilienunternehmen eines ,,professionelleren Instrumentariums" bedienten.

Schlüsselwörter: Unternehmenssteuerung · Familienunternehmen · Entscheidungsunterstützung · Verhaltenssteuerung $\cdot$ Führung

(C) Gabler-Verlag 2012

Mag. A. Posch $(\bowtie) \cdot$ Prof. Dr. G. Speckbacher

Institut für Unternehmensführung, Wirtschaftsuniversität Wien,

Nordbergstraße 15 (UZA 4), 7. Stock, Kern B, 1090 Wien, Österreich

E-Mail: arthur.posch@wu.ac.at

Prof. Dr. G. Speckbacher

E-Mail: gerhard.speckbacher@wu.ac.at 
JEL Classification: M49

\section{Motivation}

Familienunternehmen spielen sowohl in Europa als auch in den USA eine herausragende wirtschaftliche Rolle. ${ }^{1}$ Im Sinne der klassischen Prinzipal-Agenten-Theorie gelten Familienunternehmen als Organisationsform mit besonders niedrigen Agency-Kosten für die Unternehmensüberwachung (vgl. Fama und Jensen 1983; Daily und Dollinger 1992). Wenn eine Eigentümerfamilie die Mehrheit der Eigentumsrechte am Unternehmen hält, können Eigentumsrechte effektiver wahrgenommen werden und wenn darüber hinaus auch noch Familienmitglieder selbst als Mitglieder der Geschäftsführung aktiv sind, scheint eine unmittelbare Übertragung von Eigentümerinteressen in Geschäftsführungsentscheidungen gewährleistet.

Andererseits wurde darauf hingewiesen, dass diesen Vorteilen der Leitungsstruktur (Corporate Governance) von Familienunternehmen auch gewichtige Nachteile gegenüberstehen. Unternehmen, die sich mehrheitlich im Besitz einer Familie befinden, werden weniger über den Kapitalmarkt kontrolliert und diszipliniert. Wenn die Unternehmensgeschäfte zudem durch Familienmitglieder geführt werden, ist neben der geringeren Disziplinierung durch den Kapitalmarkt auch eine geringere Disziplinierung der Geschäftsführung durch den „Arbeitsmarkt für Manager“ zu erwarten (vgl. Fama und Jensen 1983). Gerade für leitende Positionen könnten Familienbande eine wichtigere Rolle spielen als die Qualifikation und bei Managementfehlern könnten Familienbande einen Schutz vor Entlassung bieten (vgl. Schulze et al. 2001). Daher wird vermutet, dass die Anreize zu rationalem und professionellem Verhalten der Geschäftsführung geringer sind und „Selbstkontrollprobleme“ sowie intuitives und zu wenig kritisch reflektiertes Entscheidungsverhalten wahrscheinlicher als in Nicht-Familienunternehmen sind (z. B. Schulze et al. 2001). Es wird aber nicht nur argumentiert, dass die Geschäftsführung in Familienunternehmen weniger Gebrauch von Entscheidungsunterstützungsinstrumenten zur Sicherung rationaler Entscheidungen macht und sich stattdessen eher auf „Bauchentscheidungen“ verlässt, sondern es wird in ähnlicher Weise argumentiert, dass in Familienunternehmen auch die Steuerung des Verhaltens von Mitarbeiter/inne/n weniger, ,professionell“"erfolgt (vgl. z. B. McEachern 1978; Schulze et al. 2001). ${ }^{2}$ Diese Besonderheiten beim Einsatz von Instrumenten zur Entscheidungsunterstützung des Top-Managements und zur Verhaltenssteuerung werden häufig als Ausdruck der geringeren Professionalität von Familienunternehmen und der geringeren Rationalität und Objektivität der Entscheidungsfindung gesehen und zumindest implizit als ein Faktor interpretiert, der den wirtschaftlichen Erfolg negativ beeinflusst (vgl. z. B. McEachern 1978; Schulze et al. 2001; Burkart et al. 2003; Hall und Nordqvist 2008).

Allerdings wird in der Literatur zu Familienunternehmen auch argumentiert, dass eine intuitivere Entscheidungsfindung und Besonderheiten bei der Verhaltenssteuerung nicht einfach auf mangelnde Professionalität im Management zurückgeführt werden können. Mintzberg und Waters (1982) weisen darauf hin, dass unternehmerische Einsichten und Visionen oft eher intuitiv als rational begründet sind und zumeist nicht (z. B. durch Kennzahlen und Berechnungen) explizit gemacht werden können. Intuitives Entscheidungsverhalten könnte vielleicht sogar ein Erfolgsfaktor für Familienunternehmen sein, weil 
hier leichter unternehmerische Entscheidungen getroffen werden können, deren Sinnhaftigkeit nicht im Vorhinein durch konkrete Zahlen und Daten gegenüber Außenstehenden (Kapitalgebern, Aufsichtsorganen) belegt werden kann. Familiäre Beziehungen können zudem als wertvolle Ressource (,soziales Kapital“) interpretiert werden, die für Familienunternehmen einen wichtigen Wettbewerbsvorteil bedeutet (vgl. Habbershon und Williams 1999; Chrisman et al. 2005; Sirmon und Hitt 2003). Insbesondere ermöglichen familiäre Beziehungen die interpersonale Übertragung impliziten Wissens über Unternehmensziele und über unternehmerische Einsichten, welches nicht explizit durch messbare Größen oder Zielvorgaben kommuniziert werden kann. Durch die Mitwirkung von Familienmitgliedern in der Geschäftsführung können derartige implizite Informationen und Einsichten in der Unternehmensführung Berücksichtigung finden und diese impliziten Einsichten und Informationen können Mitarbeiter/inne/n womöglich sogar besser durch ständige persönliche Interaktion nahegebracht werden als durch explizite Steuerungsinstrumente, wie Kennzahlen, Verhaltensvorgaben oder Anreizsysteme (vgl. Speckbacher und Wentges 2011).

Der vorliegende Beitrag untersucht anhand einer empirischen Studie, ob sich die Dominanz einer Gründerfamilie in der Eigentümerstruktur und in der Geschäftsführung auf die Nutzung von Instrumenten zur Entscheidungsunterstützung und Rationalitätssicherung der Geschäftsführung einerseits und auf die Verhaltenssteuerung andererseits auswirkt. Im Sinne des kontingenztheoretischen Ansatzes der Unternehmenssteuerung (vgl. Chenhall 2003; Donaldson 2001) wird damit die Eigentümer- und Leitungsstruktur von Unternehmen (als Charakteristikum der Corporate Governance) als Kontingenzfaktor für die Ausgestaltung von Steuerungssystemen gesehen. Zudem wird untersucht, ob Familienunternehmen, die mehr Gebrauch von Daten und Kennzahlen zur Entscheidungsunterstützung machen, tendenziell erfolgreicher (oder weniger erfolgreich) sind. Ebenso wird untersucht, ob Familienunternehmen, die sich eines ,professionelleren Instrumentariums“ der Verhaltenssteuerung bedienen, erfolgreicher sind. Hieraus können dann Hinweise zur Beantwortung der Frage abgeleitet werden, ob die häufig als „mangelnde Professionalität" von Familienunternehmen interpretierten Besonderheiten bei der Entscheidungsfindung und bei der Verhaltenssteuerung tatsächlich ein Nachteil sind. Unsere empirische Untersuchung basiert auf Befragungsdaten von 230 kleinen und mittleren Produktionsunternehmen (50-250 Mitarbeiter/innen) im deutschsprachigen Raum.

\section{Besonderheiten der Führung von Familienunternehmen}

Wenngleich keine in der einschlägigen Literatur einheitlich zugrunde gelegte Definition von Familienunternehmen existiert, haben die meisten Definitionen gemeinsam, dass als wesentliches Charakteristikum von Familienunternehmen die dominante Rolle einer (Gründer-) Familie bei der Festlegung der Unternehmensvision und der Unternehmensziele sowie in der Führung und Steuerung des Unternehmens gesehen wird (vgl. Sharma 2004). Unter den in der Literatur verwendeten Kriterien für die konkrete Identifikation und Abgrenzung von Familienunternehmen kommt den beiden Kriterien „Unternehmen ist überwiegend in Familienbesitz“ und „Dominanter Einfluss von Familienmitgliedern in der Geschäftsführung“ die wichtigste Bedeutung zu. Dabei wird argumentiert, dass Besonderheiten der Führung vor allem dann auftreten, wenn das Unternehmen nicht nur 
überwiegend im Besitz der Gründerfamilie ist, sondern wenn zusätzlich die Geschäftsführung überwiegend aus Mitgliedern der Gründerfamilie besteht (insbesondere dann, wenn der CEO Familienmitglied ist) bzw. wenn es sich um rein eigentümergeführte Unternehmen handelt (vgl. Chua et al. 1999; Daily und Dollinger 1992; Kotey 2005; Minichilli et al. 2010; Speckbacher und Wentges 2011; Tagiuri und Davis 1996). Neben dem Kriterium des überwiegenden Familienbesitzes wird im Folgenden daher der dominante Einfluss der Gründerfamilie in der Geschäftsführung als Abgrenzungsmerkmal zugrunde gelegt, wobei dominant hier sehr eng als ,,ausschließlich aus Familienmitgliedern bestehende Geschäftsführung“ interpretiert wird (,eigentümergeführtes Unternehmen“).

\subsection{Entscheidungsfindung der Geschäftsführung von Familienunternehmen}

Mintzberg und Waters (1982) argumentieren, dass unternehmerische Einsichten und Visionen oft auf Intuition und implizitem Wissen basieren und daher kaum in Form expliziter strategischer Pläne, Messgrößen und Kennzahlen formuliert werden können. Daher sei zu erwarten, dass eigentümergeführte Unternehmen bei Entscheidungen eher intuitiv handeln und weniger auf Entscheidungsrechnungen und Kennzahlen zurückgreifen. Daily und Dollinger (1992) argumentieren in ähnlicher Weise, dass in Familienunternehmen weniger Bedarf dafür besteht, Rechenschaft gegenüber fremden und in die Unternehmensgeschäfte nicht involvierten Eigentümern abzulegen. Daher können Entscheidungsprozesse weniger formal ablaufen. Schulze et al. (2001) argumentieren hingegen, dass bei der Entscheidungsfindung in Familienunternehmen "Selbstkontrollprobleme“ eine besondere Rolle spielen, d. h. Entscheidungen werden oft nicht kritisch reflektiert unter Zuhilfenahme von Entscheidungsrechnungen und Kennzahlen getroffen, sondern eher ,aus dem Bauch heraus". Während die in der Corporate Governance typischerweise thematisierten Probleme der Kontrolle der Geschäftsführung durch die Eigentümer in Familienunternehmen (die überwiegend im Familienbesitz sind und durch Familienangehörige geführt werden) demnach eine wesentlich geringere Rolle spielen, sind hier „Selbstkontrollprobleme“ von wesentlich höherer Bedeutung. Als entscheidender Grund hierfür wird die mangelnde Disziplinierung der Geschäftsführung von Familienunternehmen durch den Kapitalmarkt gesehen sowie die Bestellung von Geschäftsführern auf Grund der Familienzugehörigkeit anstatt der rein fachlichen Qualifikation. Geschäftsführer, die der Gründerfamilie angehören, werden auch weniger durch den Arbeitsmarkt für Manager diszipliniert (also durch andere Manager, die mit ihnen um die Geschäftsführerposition konkurrieren) und wegen der Bedeutung der Familienzugehörigkeit als Einstellungskriterium ist es wahrscheinlicher, dass die fachliche Qualifikation für die Anwendung professioneller Instrumente zur Entscheidungsunterstützung fehlt. Damit lehnen sich Schulze et al. (2001) an eine Reihe früherer Arbeiten an (vgl. z. B. McEachern 1978; Chandler 1977, 1990), die in Familienunternehmen ein weniger professionelles und weniger rational-reflektiertes Entscheidungsverhalten der Geschäftsführung vermuten. Beide in der Literatur zu findenden Argumentationslinien zu den Besonderheiten im Entscheidungsverhalten der Geschäftsführung von Familienunternehmen kommen damit - wenn auch mit sehr unterschiedlichen Argumenten - zur selben Schlussfolgerung, wonach die Geschäftsführung von Familienunternehmen Entscheidungen eher intuitiv trifft und weniger auf „objektive“ Daten und Kennzahlen zur Entscheidungsunterstützung zurückgreift als die Geschäftsführung von Nicht-Familienunternehmen. 
H1: Auf Geschäftsführungsebene machen Familienunternehmen weniger Gebrauch von Daten und Kennzahlen zur Entscheidungsunterstützung als Nicht-Familienunternehmen.

\subsection{Verhaltenssteuerung in Familienunternehmen}

Im Rahmen der kontingenztheoretischen Forschung wurde eine Reihe von Einflussfaktoren untersucht, von denen die Gestaltung von Verhaltenssteuerungssystemen in Unternehmen abhängt. Als wesentliche Einflussfaktoren wurden hierbei die Unternehmensgröße, die Organisationsstruktur, die Unternehmensstrategie, die Umfeldunsicherheit und die Unternehmenstechnologie untersucht (vgl. Chenhall 2003; Donaldson 2001). Welchen Einfluss „Governance-Charakteristika“, wie etwa Eigenschaften der Eigentümerstruktur oder der Geschäftsführung, auf die Art und Weise haben, wie die Geschäftsführung nachgelagerte Manager oder Mitarbeiter/innen steuert, wurde bisher erst in Ansätzen untersucht (vgl. Geeraerts 1984; Davila 2005; Davila und Foster 2007; Speckbacher und Wentges 2011). Zur Klassifikation von Verhaltenssteuerungsinstrumenten soll im Folgenden die auf Ouchi (1979) aufbauende Klassifikation von Steuerungsinstrumenten nach Merchant und Van der Stede (2007) verwendet werden. Hierbei werden Steuerungsinstrumente danach klassifiziert, welches „Steuerungsobjekt“ dem jeweiligen Instrument zugrunde liegt. Steuerungsinstrumente können demnach 1) unmittelbar an der Aufgabenverrichtung der Mitarbeiter/innen ansetzen und direkt versuchen, durch Vorgaben oder Verhaltensüberwachung erwünschtes Verhalten sicherzustellen (,action controls“), sie können 2) an den Ergebnissen der Mitarbeitertätigkeit ansetzen, also beispielsweise durch Kennzahlen zur Beurteilung der Zielerreichung Mitarbeiterverhalten beeinflussen (,results controls“), sie können 3) an der Organisationskultur ansetzen und versuchen, das Verhalten von Mitarbeiter/inne/n durch Etablieren einer geeigneten Organisationskultur zu beeinflussen (,,cultural controls“) und sie können 4) an der Person ansetzen, d. h. bereits bei der Mitarbeiter/innen/auswahl und bei der Schulung und Entwicklung der Mitarbeiter/innen (,,personnel controls“). Der vorliegende Beitrag konzentriert sich auf die Verhaltenssteuerung im engeren Sinne und klammert Fragen der Personalauswahl und -entwicklung aus.

Wenngleich eine umfassende, theoriegeleitete Untersuchung der Besonderheiten der Verhaltenssteuerung in Familienunternehmen bisher fehlt, enthält die Literatur zu Familienunternehmen eine Reihe von Argumenten, die untermauern, dass sich (eigentümergeführte) Familienunternehmen bei der Verhaltenssteuerung von anderen Unternehmen unterscheiden. Insgesamt wird in der Literatur zu Familienunternehmen recht einheitlich argumentiert, dass sich die Geschäftsführung von Familienunternehmen im Vergleich zu Nicht-Familienunternehmen anderer Mechanismen bei der Verhaltenssteuerung bedient.

Tagiuri und Davis (1996, S. 201) charakterisieren Familienunternehmen durch die Überlappung der drei Sphären „Familie“, „Eigentum“ und „Geschäftsführung“ und leiten daraus eine informellere, auf persönlichen Beziehungen basierende Führung und Verhaltenssteuerung $\mathrm{ab}$. In ähnlicher Weise argumentieren Daily und Dollinger (1992), dass die Geschäftsführung in Familienunternehmen wegen des geringeren Bedarfs, Rechenschaft gegenüber Externen abzulegen und wegen der geringeren Bedeutung von AgencyKonflikten bei der Führung und Steuerung von Mitarbeiter/inne/n weniger formalisierte Steuerungssysteme einsetzt und dass eine informelle, persönliche Steuerung im Rahmen der Sphäre „Familie“ die in anderen Unternehmen üblichen formalen Steuerungssysteme zumindest teilweise ersetzt (ähnlich auch Flamholtz 1986). 
Jorissen et al. (2005) verweisen auf die Überlappung der Eigentums- und Führungsrollen und die sich daraus ergebende geringere Notwendigkeit von ergebnisbezogener Steuerung in Familienunternehmen. Zudem ist eine „faire“, rein kennzahlenorientierte Leistungsbeurteilung und Steuerung schlecht vereinbar mit einer besonderen Behandlung von Familienmitgliedern (Jorissen et al. 2005). Eine kennzahlenorientierte Steuerung setzt zudem ein Instrumentenwissen voraus, über das Geschäftsführer von Familienunternehmen tendenziell seltener verfügen (Jorissen et al. 2005). Ähnlich argumentieren Schulze et al. (2001), dass Familienunternehmen bei der Verhaltenssteuerung wegen des fehlenden Marktdruckes weniger in ergebnisorientierte Überwachungs- und Anreizsysteme investieren und weniger ergebnisorientiert entlohnen.

H2a: Bei der Verhaltenssteuerung auf Mitarbeiterebene machen Familienunternehmen in geringerem Umfang Gebrauch von Kennzahlen zur ergebnisorientierten Steuerung als Nicht-Familienunternehmen.

Die Literatur zu Familienunternehmen betont nahezu einheitlich, dass die Entscheidungsstrukturen in Familienunternehmen stärker zentralisiert sind, wodurch eine größere direkte Einflussnahme der Geschäftsführung auf die Aufgabenverrichtung erfolgt (Dyer 1986; Geeraerts 1984; Daily und Dollinger 1992). Nach Dyer (1986) haben familiengeführte Unternehmen oft ,,paternalistische“ Führungsstrukturen mit stark zentralisierten Entscheidungsstrukturen. Ähnlich charakterisieren auch Harris et al. (2004) Familienunternehmen als stark zentralisiert mit einer großen Bedeutung von direkten Verhaltensvorgaben und -eingriffen. Kets de Vries (1993) verweist darauf, dass Familienunternehmen oftmals von einer unternehmerischen Persönlichkeit geprägt werden, die eine Präferenz für zentrale Entscheidungsprozesse und die Anwendung von Verhaltensvorgaben hat. Da EigentümerManager oft ihr eigenes Vermögen im Unternehmen konzentriert haben, besteht auch ein größeres Bedürfnis, Entscheidungsmacht zu zentralisieren und Mitarbeiter/innen an Verhaltensvorgaben zu binden anstatt auf Autonomie und Delegation zu setzen (Daily und Dollinger 1992).

$H 2 b$ : Familienunternehmen sind durch einen geringeren diskretionären Entscheidungsspielraum von Mitarbeiter/inne/n charakterisiert als Nicht-Familienunternehmen.

Zahra et al. (2004) argumentieren, dass in Familienunternehmen die Organisationskultur als informeller Steuerungsmechanismus formale Steuerungsmechanismen ersetzen kann. Allerdings bleibt dabei unklar, ob Familienunternehmen gemeinsame Werte und Verhaltensnormen lediglich implizit (beispielsweise im Sinne einer Vorbildfunktion) einsetzen oder auch explizit formulieren und kommunizieren. Mintzberg und Waters (1982) illustrieren anhand von Fallbeispielen, dass Familienunternehmen persönliche Interaktion und persönliche Beziehungen anstatt formaler Instrumente zur Implementierung von Strategien einsetzen. Demnach werden Ziele und Normen in eigentümergeführten Unternehmen oft informell durch die Omnipräsenz der Eigentümer-Geschäftsführer im Unternehmen implementiert. In ähnlicher Weise betonen Habbershon und Williams (1999) die Bedeutung des durch die Unternehmensgründer geprägten Wertesystems und legen nahe, dass die Kultur in Familienunternehmen eher durch implizite Mechanismen, wie etwa die Vorbildfunktion der Unternehmensgründer oder der Gründerfamilie, geprägt ist. Wird also angenommen, dass die Vermittlung der Unternehmenskultur an die Mitarbeiter/innen überwiegend auf 
implizitem Wege durch die aktive Mitwirkung der Familienmitglieder am täglichen Geschäft des Unternehmens erfolgt, so ist zu erwarten, dass die explizite Formulierung und Kommunikation von Werten und Verhaltensnormen in Form von „Mission Statements“ oder „Codes of Conduct“ in Familienunternehmen eine geringere Rolle spielt.

H2c: Zur Steuerung des Mitarbeiterverhaltens setzen Familienunternehmen weniger auf explizit formulierte und kommunizierte Werte und Verhaltensnormen als NichtFamilienunternehmen.

\subsection{Erfolgswirkungen des Einsatzes von Instrumenten zur Entscheidungsunterstützung und zur Verhaltenssteuerung}

Wie oben dargestellt, wird in der Literatur zu Familienunternehmen von einer Reihe von Autoren die Auffassung vertreten, dass Familienunternehmen sowohl bei der Entscheidungsfindung als auch bei der Verhaltenssteuerung weniger professionell agieren als NichtFamilienunternehmen, wodurch verschiedene Instrumente weniger zum Einsatz kommen (vgl. McEachern 1978; Schulze et al. 2001; Burkart et al. 2003; Hall und Nordqvist 2008). Als Gründe für die geringere Professionalität werden im Wesentlichen der in Familienunternehmen geringere Druck des Kapitalmarktes, Entscheidungen zu rechtfertigen und professionelle „State-of-the-Art Methoden“ bei der Entscheidungsfindung und der Verhaltenssteuerung einzusetzen genannt sowie die Besetzung von Führungspositionen nach Familienzugehörigkeit anstatt einer rein von der Qualifikation abhängigen Besetzung. Letzteres führt gemäß dieser Argumentation dazu, dass Top-Manager in Familienunternehmen tendenziell schlechter qualifiziert sind und einem geringeren Erfolgsdruck ausgesetzt sind, woraus dann auf geringere „Professionalität“ geschlossen wird. Zumindest implizit wird hierbei unterstellt, dass Familienunternehmen erfolgreicher sein könnten, wenn sie bei der Entscheidungsfindung und bei der Verhaltenssteuerung ,professioneller“ agierten. Daraus ließe sich die Erwartung ableiten, dass Familienunternehmen, die mehr Gebrauch von Daten und Kennzahlen zur Entscheidungsunterstützung und zur Verhaltenssteuerung machen - bzw. sich beim Einsatz von Instrumenten zur Verhaltenssteuerung ähnlich wie Nicht-Familienunternehmen verhalten (also mehr Kennzahlen zur ergebnisorientierten Steuerung einsetzen, ihren Mitarbeiter/inne/n höheren Entscheidungsspielraum gewähren und stärker durch explizit formulierte und kommunizierte Werte und Normen steuern) - erfolgreicher sind.

H3: Familienunternehmen, die auf Geschäftsführungsebene mehr Gebrauch von Daten und Kennzahlen zur Entscheidungsunterstützung machen, sind tendenziell erfolgreicher als andere Familienunternehmen.

H4a: Familienunternehmen, die zur Verhaltenssteuerung auf Mitarbeiterebene mehr Gebrauch von Kennzahlen zur ergebnisorientierten Steuerung machen, sind erfolgreicher als andere Familienunternehmen.

H4b: Familienunternehmen, die ihren Mitarbeiter/inne/n einen höheren diskretionären Entscheidungsspielraum gewähren, sind erfolgreicher als andere Familienunternehmen.

H4c: Familienunternehmen, die zur Verhaltenssteuerung auf Mitarbeiterebene mehr auf explizit formulierte und kommunizierte Werte und Verhaltensnormen setzen, sind erfolgreicher als andere Familienunternehmen. 


\section{Vorgehen bei der Datensammlung und -auswertung}

Zur Datenerhebung wurde ein strukturierter Online-Fragebogen verwendet. Dazu wurden aus der Grundgesamtheit aller in der Markus Datenbank (Bureau van Dijk Electronic Publishing) enthaltenen deutschen und österreichischen Produktionsunternehmen mit 50-250 Mitarbeiter/inne/n 1400 Unternehmen zufällig ausgewählt, von denen im Winter 2008/Frühjahr 2009 schließlich 1357 Unternehmen per E-Mail kontaktiert werden konnten (fehlende E-Mail Kontaktdaten bei 43 Unternehmen). Der Fragebogen wurde jeweils an die Geschäftsführung der Unternehmen geschickt. Aufgrund mehrmaliger Erinnerungsschreiben konnten schließlich 230 ausgefüllte Fragebögen ausgewertet werden (Antwortrate 16,9\%).

Der Fragebogen besteht insgesamt aus 90 Fragen. Zur Unterscheidung von Familienunternehmen und Nicht-Familienunternehmen wurde abgefragt, zu welchem Anteil das jeweilige Unternehmen im Besitz der Gründerfamilie ist und ob Mitglieder der Gründerfamilie die Geschäftsführung stellen. Unternehmen, die überwiegend ( $>50 \%)$ in Besitz der Gründerfamilie sind und deren Geschäftsführung ausschließlich aus Mitgliedern der Gründerfamilie besteht, wurden als Familienunternehmen bzw. als familiengeführte Unternehmen klassifiziert.

Alle zentralen Konstrukte (Entscheidungsverhalten, Verhaltenssteuerungsinstrumente) wurden durch jeweils 3-4 Fragen operationalisiert, und für jede Frage wurde eine 7-Punkt-Skala zugrundegelegt ( 1 = nicht zutreffend und 7 = völlig zutreffend). Die Konstrukte zur Charakterisierung von Verhaltenssteuerungssystemen wurden weitgehend aus der Literatur entnommen (vgl. Widener 2007; Henri 2006; s. Anhang). Für die Erfassung des Entscheidungsverhaltens der Geschäftsführung (intuitiv vs. Verwendung von Instrumenten zur Entscheidungsunterstützung) wurde ein eigenes Konstrukt entwickelt. Zur Messung des Unternehmenserfolges wurde ein Perzeptionsmaß verwendet, d. h. eine Einschätzung der Befragten über den Unternehmenserfolg im Vergleich zu den Mitbewerbern (vgl. Anhang). Die für die einzelnen reflexiven Konstrukte verwendeten Items wurden zunächst einer explorativen Faktorenanalyse unterzogen. Neben dieser explorativen Faktorenanalyse wurde auch eine konfirmatorische Faktorenanalyse durchgeführt und die Qualität des Messmodells anhand der Kriterien Cronbach Alpha, Faktorreliabilität und durchschnittlich erfasste Varianz evaluiert. Zudem wurden die wichtigsten, in der Kontingenzforschung zu Verhaltenssteuerungssystemen (vgl. z. B. Chenhall 2003) identifizierten Einflussfaktoren der Gestaltung von Verhaltenssteuerungssystemen als Kontrollvariablen erfasst: Die Unternehmensgröße wurde mit der Anzahl der Mitarbeiter/innen gemessen, ferner wurde das Unternehmensalter als Kontrollvariable verwendet (vgl. Moores und Mula 2000; Moores und Yuen 2001). Die strategische Ausrichtung des Unternehmens wurde über die Innovationsorientierung (Explore/Exploit) gemessen (vgl. Zahra et al. 2000), bei der Messung der Umfeldunsicherheit wurde auf ein etabliertes Konstrukt von Moers (2006) zurückgegriffen (Tab. 1).

Um das Ausmaß eines möglichen Non-Response-Bias zu bestimmen, wurde in Anlehnung an Armstrong und Overton (1977) ein Vergleich zwischen früh und spät antwortenden Unternehmen auf Itemebene durchgeführt. Die Mittelwertvergleiche ergaben keine Hinweise auf einen wesentlichen Non-Response-Bias. 
Tab. 1: Güte der Faktoren

\begin{tabular}{llllll}
\hline Faktor & $\begin{array}{l}\text { Anzahl } \\
\text { der Items }\end{array}$ & $\begin{array}{l}\text { Spannbreite } \\
\text { der Ladungen }\end{array}$ & $\begin{array}{l}\text { Cronbach's } \\
\text { Alpha }\end{array}$ & $\begin{array}{l}\text { Faktorreli- } \\
\text { abilität }\end{array}$ & $\begin{array}{l}\text { Durchschnittlich } \\
\text { erfasste Varianz }\end{array}$ \\
\hline Action controls & 3 & $0,571-0,899$ & 0,673 & 0,88 & 0,63 \\
Results controls & 3 & $0,884-0,921$ & 0,875 & 0,97 & 0,81 \\
Cultural controls & 4 & $0,618-0,935$ & 0,830 & 0,96 & 0,72 \\
Rationalitätssicherung & 3 & $0,657-0,863$ & 0,707 & 0,89 & 0,64 \\
Umfeldunsicherheit & 4 & $0,781-0,844$ & 0,811 & 0,96 & 0,64 \\
Exploration & 4 & $0,580-0,873$ & 0,754 & 0,92 & 0,59 \\
Exploitation & 3 & $0,704-0,801$ & 0,615 & 0,89 & 0,55 \\
Unternehmenserfolg & 3 & $0,886-0,924$ & 0,891 & 0,98 & 0,82 \\
\hline
\end{tabular}

In Anlehnung an ähnlich geartete frühere Studien (vgl. z. B. Carrasco-Hernandez und Sanchez-Marin 2007; Kotey 2005) wurden ANCOVA-Analysen durchgeführt, um auf Unterschiede zwischen Familien- und Nichtfamilienunternehmen zu testen (vgl. Bracker et al. 1988; Garson 2008; Hair et al. 2006). Die für die ANCOVA notwendigen Voraussetzungen (Hair et al. 2006) der Daten wurden überprüft und werden hinreichend erfüllt. ${ }^{3}$ Zur Untersuchung von Hypothesen drei und vier werden multivariate OLS-Regressionen verwendet, deren Anwendung für die Untersuchung von Performanceeffekten in bisheriger Management Control-Forschung weit verbreitet ist (Chenhall 2003; Hartmann und Moers 1999). Die Überprüfung der notwendigen Datenvoraussetzungen bestätigt die Anwendbarkeit der gewählten Methode. ${ }^{4}$

\section{Ergebnisse}

Hypothese 1 postuliert, dass Geschäftsführer von Familienunternehmen Instrumente zur Entscheidungsunterstützung in geringerem Ausmaß verwenden als Nicht-Familienunternehmen. Der Test dieser Hypothese erfolgt mittels einer Kovarianzanalyse. Im Rahmen dieser Analyse werden neben dem Faktor (Familien- vs. Nichtfamilienunternehmen) auch Kovariate/Kontrollvariable berücksichtigt (Größe, Alter, strategische Ausrichtung und Umfeldunsicherheit), um deren Einfluss zu bereinigen (vgl. Backhaus et al. 2003; Garson 2008) (Tab. 2).

Die Ergebnisse der Analyse zeigen, dass Nicht-Familienunternehmen $($ Mittelwert $=$ 4,61; Standardabweichung =1,12) im Vergleich zu Familienunternehmen (Mittelwert = 3,95; Standardabweichung $=1,32$ ) signifikant stärker Instrumente zur Entscheidungsunterstützung in der Geschäftsführung einsetzen $(F=14,428 ; p<0,01)$. Einen ebenfalls signifikanten Einfluss zeigen die Variablen Umfeldunsicherheit $(F=12,009 ; p<0.01)$ sowie Exploitation $(\mathrm{F}=3,963 ; \mathrm{p}<0.05)$.

Interessanterweise zeigt sich kein signifikanter Einfluss von Unternehmensgröße auf die Verwendung der untersuchten Steuerungsinstrumente. Dies widerspricht zwar Ergebnissen bisheriger kontingenztheoretischer Forschung, die einen Einfluss der Unternehmensgröße auf die Verwendung von Steuerungssystemen belegen (vgl. Chenhall 2003), lässt sich aber dadurch erklären, dass alle untersuchten Unternehmen relativ klein sind (50-250 Mitarbeiter/innen). 
Tab. 2: Deskriptive Statistik für Instrumente der Unternehmenssteuerung

\begin{tabular}{llc}
\hline Instrumente der Unternehmenssteuerung & \multicolumn{2}{l}{ Nicht-Familienunternehmen, Familienunternehmen, } \\
& $\begin{array}{l}\text { N=132; Mittelwert } \\
\text { (Standardabweichung) }\end{array}$ & $\begin{array}{l}\text { N=98; Mittelwert } \\
\text { (Standardabweichung) }\end{array}$ \\
\hline $\begin{array}{l}\text { Entscheidungsunterstützung } \\
\text { Rationalitätssicherung in der } \\
\text { Geschäftsführung (Selbstkontrolle) }\end{array}$ & $4,61(1,12)$ & $3,95(1,32)$ \\
$\begin{array}{l}\text { Verhaltenssteuerung der Mitarbeiter/innen } \\
\text { Steuerung der Aufgabenverrichtung } \\
\text { (action controls) }\end{array}$ & $2,65(1,03)$ & $3,15(1,32)$ \\
$\begin{array}{l}\text { Steuerung über Ergebnisse } \\
\text { (results controls) }\end{array}$ & $5,67(1,06)$ & $5,13(1,33)$ \\
Kulturelle Steuerung (cultural controls) & $4,92(1,19)$ & $4,48(1,40)$ \\
\hline
\end{tabular}

Tab. 3: Ergebnisse der ANCOVA für $\mathrm{H} 1$

\begin{tabular}{lcc}
\hline Faktor/Kovariate & $\mathrm{F}-$ Wert & $\mathrm{p}$-Wert \\
\hline Familien- vs. Nicht- & $14,428^{* * *}$ & 0,000 \\
Familienunternehmen & & \\
Unternehmensgröße & 0,119 & 0,731 \\
Unternehmensalter & 0,863 & 0,354 \\
Exploration & 0,004 & 0,947 \\
Exploitation & $3,963^{* *}$ & 0,048 \\
Umfeldunsicherheit & $12,009^{* * *}$ & 0,001 \\
${ }^{* * *} \mathrm{p}<0,01{ }^{* *} \mathrm{p}<0,05$ & &
\end{tabular}

Konsistent mit den angeführten Argumentationslinien zeigt sich, dass Familienunternehmen von einem eher intuitiv-geprägten Entscheidungsverhalten in der Geschäftsführung Gebrauch machen (Tab. 3).

Zum Test von Hypothesen 2abc, wonach ein signifikanter Unterschied zwischen Familien- und Nicht-Familienunternehmen hinsichtlich des Einsatzes von Instrumenten der Verhaltenssteuerung besteht, erfolgt die Berechnung von univariaten Kovarianzanalysen (ANCOVA) (d. h. für jedes Instrument zur Verhaltenssteuerung wird eine separate Analyse durchgeführt). Als Kovariaten fungieren neuerlich Unternehmensgröße, Alter, strategische Ausrichtung und Umfeldunsicherheit.

Der Klassifikation von Merchant und Van der Stede (2007) folgend werden folgende Instrumente der Verhaltenssteuerung als abhängige Variablen untersucht: Steuerung der Aufgabenverrichtung (action controls), Steuerung über Ergebnisse (results controls), kulturelle Steuerung (cultural controls).

Die Analyseergebnisse zeigen, dass Familienunternehmen (Mittelwert=4,48; Standardabweichung = 1,40) im Vergleich zu Nicht-Familienunternehmen (Mittelwert=4,92; Standardabweichung $=1,19)$ signifikant weniger Gebrauch machen von kulturellen Steuerungsinstrumenten $(\mathrm{F}=6,169 ; \mathrm{p}<0,05)$.

Ebenso zeigt sich, dass in Nicht-Familienunternehmen (Mittelwert = 5,67; Standardabweichung $=1,06$ ) Mitarbeiter/innen signifikant stärker anhand von Kennzahlen/Resultaten beurteilt werden $(\mathrm{F}=11,411 ; \mathrm{p}<0,01)$ als in Familienunternehmen (Mittelwert = 5,13; Standardabweichung $=1,33$ ). 
Tab. 4: Ergebnisse der ANCOVA für H2abc

\begin{tabular}{llll}
\hline Faktor/Kovariate & $\begin{array}{l}\text { Steuerung der } \\
\text { Aufgabenverrichtung } \\
\text { (action controls) }\end{array}$ & $\begin{array}{l}\text { Steuerung über } \\
\text { Ergebnisse (results } \\
\text { controls) }\end{array}$ & $\begin{array}{l}\text { Kulturelle Steuerung } \\
\text { (cultural controls) }\end{array}$ \\
\hline Familien- vs. Nicht- & $F=9,346^{* * *}$ & $F=11,411^{* * *}$ & $F=6,169^{* *}$ \\
Familienunternehmen & $p=0,003$ & $p=0,001$ & $p=0,014$ \\
Unternehmensgröße & $\mathrm{F}=2,016$ & $\mathrm{~F}=0,186$ & $\mathrm{~F}=0,023$ \\
& $\mathrm{p}=0,157$ & $\mathrm{p}=0,666$ & $\mathrm{p}=0,880$ \\
Unternehmensalter & $\mathrm{F}=2,168$ & $\mathrm{~F}=0,946$ & $\mathrm{~F}=0,097$ \\
& $\mathrm{p}=0,142$ & $\mathrm{p}=0,332$ & $\mathrm{p}=0,755$ \\
Exploration & $\mathrm{F}=2,051$ & $\mathrm{~F}=1,031$ & $\mathrm{~F}=1,537$ \\
& $\mathrm{p}=0,153$ & $\mathrm{p}=0,311$ & $\mathrm{p}=0,216$ \\
Exploitation & $\mathrm{F}=1,433$ & $F=11,851^{* * *}$ & $F=8,297^{* * *}$ \\
& $\mathrm{p}=0,233$ & $p=0,001$ & $p=0,004$ \\
Umfeldunsicherheit & $\mathrm{F}=0,103$ & $F=3,048^{*}$ & $\mathrm{~F}=0,434$ \\
& $\mathrm{p}=0,748$ & $p=0,082$ & $\mathrm{p}=0,511$ \\
\hline
\end{tabular}

${ }^{* * *} \mathrm{p}<0,01 ;{ }^{* *} \mathrm{p}<0,05 ;{ }^{*} \mathrm{p}<0,1$

Eine Steuerung der Aufgabenverrichtung hingegen findet in Familienunternehmen (Mittelwert $=3,15$; Standardabweichung $=1,32)$ eine signifikant stärkere Verwendung $(\mathrm{F}=$ 9,346; $\mathrm{p}<0,01$ ) als in Nicht-Familienunternehmen (Mittelwert=2,65; Standardabweichung=1,03).

Während die Kontrollvariablen Unternehmensgröße und Unternehmensalter keinen signifikanten Einfluss auf die Verwendung von Steuerungsinstrumenten zeigen, sind bei den Variablen Umfeldunsicherheit und strategische Ausrichtung teilweise signifikante Effekte zu beobachten (Tab. 4).

Im letzten Teil der Analyse wird der Zusammenhang zwischen der Verwendung von Steuerungsinstrumenten in Familienunternehmen und deren Performance (gemessen durch ein Perzeptionsmaß) näher betrachtet. Der Zusammenhang zwischen dem Einsatz von Steuerungsinstrumenten zur Entscheidungsunterstützung und zur Verhaltenssteuerung und dem Erfolg von Familienunternehmen wird mithilfe einer OLS-Regressionsanalyse näher untersucht.

Das Regressionsverfahren dient dabei zur Analyse von Beziehungen zwischen einer abhängigen Variablen (Unternehmenserfolg) und mehreren unabhängigen Variablen (Einsatz der Steuerungsinstrumente) (vgl. Backhaus et al. 2003; Garson 2008; Hair et al. 2006). Als Kontrollvariablen fungieren Unternehmensgröße, Unternehmensalter und Umfeldunsicherheit.

Die Analyseergebnisse für Familienunternehmen zeigen signifikante Zusammenhänge der Performance mit der Steuerung über Ergebnisse (results controls; $\beta=0,203 ; p<0,1$ ), d. h. die Verwendung von Ergebniskontrollen für Mitarbeiter/innen hat in Familienunternehmen eine positive Auswirkung auf den Unternehmenserfolg. Ebenso einen positiven Zusammenhang mit der Unternehmensperformance zeigt die explizite Formulierung und Kommunikation von Werten und Verhaltensnormen $(\beta=0,197 ; p<0,1)$. Die Steuerung der Aufgabenverrichtung zeigt zwar wie erwartet einen negativen Zusammenhang mit der Performance $(\beta=-0,022)$, d. h. also, dass Familienunternehmen, die einen höheren diskretionären Entscheidungsspielraum gewähren, tendenziell erfolgreicher sind. Allerdings ist dieser Zusammenhang statistisch nicht signifikant und auch vom Umfang her (Regressi- 
Tab. 5: Ergebnisse der Regression für Familienunternehmen (H3, H4abc)

\begin{tabular}{|c|c|c|c|}
\hline \multirow{2}{*}{$\frac{\text { Abhängige Variable }}{\text { Unabhängige Variablen }}$} & \multicolumn{2}{|c|}{ Unternehmenserfolg } & \multirow{2}{*}{$\begin{array}{l}\text { Variance Inflation Facto } \\
\text { VIF }^{\mathrm{a}}\end{array}$} \\
\hline & $\beta$ & P-Wert & \\
\hline Konstante & $5,200^{* * *}$ & 0,000 & \\
\hline Selbstkontrolle & $-0,236^{* *}$ & 0,042 & 1,522 \\
\hline action controls & $-0,022$ & 0,822 & 1,056 \\
\hline results controls & $0,203^{*}$ & 0,065 & 1,372 \\
\hline cultural controls & $0,197^{*}$ & 0,094 & 1,563 \\
\hline Unternehmensgröße & $0,215^{* *}$ & 0,029 & 1,095 \\
\hline Unternehmensalter & $-0,071$ & 0,470 & 1,100 \\
\hline Umfeldunsicherheit & $-0,305^{* * *}$ & 0,003 & 1,161 \\
\hline $\mathrm{R}^{2}$ & \multicolumn{2}{|c|}{0,222} & \\
\hline Adjusted $\mathrm{R}^{2}$ & \multicolumn{2}{|c|}{0,162} & \\
\hline F-Wert & \multicolumn{2}{|c|}{$3,675^{* * *}$} & \\
\hline $\mathrm{N}$ & \multicolumn{2}{|c|}{98} & \\
\hline
\end{tabular}

${ }^{* * *} \mathrm{p}<0,01 ;{ }^{* *} \mathrm{p}<0,05 ;{ }^{*} \mathrm{p}<0,1$

${ }^{a} \mathrm{Da}$ die einzelnen Varianzinflationsfaktoren den Wert 5 nicht übersteigen, sollte Mulitkollinearität kein Problem darstellen (Menard 1995; Hair et al. 2006)

onskoeffizient) unbeträchtlich. Betrachtet man hingegen die Verwendung von Steuerungsinstrumenten zur Entscheidungsunterstützung in der Geschäftsführung (Selbstkontrolle), so zeigt sich ein signifikant negativer Effekt $(\beta=-0,236, p<0,05)$. Anders formuliert bedeutet dies, dass gemäß dem untersuchten Datensatz eine intuitivere Entscheidungsfindung in der Geschäftsführung von Familienunternehmen signifikant mit einem höheren Unternehmenserfolg assoziiert ist. Dies deutet also darauf hin, dass die Besonderheiten der Entscheidungsfindung in der Geschäftsführung von Familienunternehmen nicht Ausdruck mangelnder Professionalität, sondern Ausdruck einer adäquaten Anpassung an die Spezifika von Familienunternehmen sind und intuitiv geprägtes Entscheidungsverhalten sogar ein Erfolgsfaktor sein kann.

Des Weiteren ergeben sich ein signifikant positiver Effekt für Unternehmensgröße $(\beta=0,215 ; \mathrm{p}<0,05)$ und ein signifikant negativer Effekt von Umfeldunsicherheit auf den Unternehmenserfolg $(\beta=-0,305 ; \mathrm{p}<0,01)$ (Tab. 5).

\section{Zusammenfassung und Diskussion}

Unsere empirische Studie liefert Belege für folgende Zusammenhänge:

1. Familienunternehmen unterscheiden sich sowohl in ihrer Entscheidungsfindung auf Geschäftsführungsebene als auch bei der Verhaltenssteuerung auf Mitarbeiterebene signifikant von Nicht-Familienunternehmen.

2. Die intuitivere, weniger auf Daten und Kennzahlen basierende Entscheidungsfindung auf Geschäftsführungsebene geht nicht auf Kosten des Unternehmenserfolges, sondern kann sogar als Erfolgsfaktor von Familienunternehmen gesehen werden. Was gut für Nicht-Familienunternehmen ist, muss also nicht auch gut für Familienunternehmen sein. Auch hinsichtlich der Verwendung von Verhaltenssteuerungsinstrumenten ergibt sich ein differenziertes Bild. Während in unserem Sample die stärkere Zentralisierung 
von Entscheidungen in Familienunternehmen keine negative Auswirkung auf den Unternehmenserfolg hat, liefern unsere Daten Indizien, dass ein vermehrter Einsatz ergebnisorientierter Kennzahlen zur Verhaltenssteuerung sowie eine explizite Formulierung und Kommunikation von Normen und Werten in Familienunternehmen vorteilhaft sein könnte.

Unsere empirischen Ergebnisse zu den Besonderheiten von Familienunternehmen sind konsistent mit bisherigen Forschungsarbeiten. Bei der Entscheidungsfindung gehen Familienunternehmen eher intuitiv vor und Entscheidungen auf Geschäftsführungsebene werden weniger durch Entscheidungsrechnungen überprüft und gerechtfertigt. Interessant ist aber, dass sich dies keineswegs negativ auf den Erfolg von Familienunternehmen auswirkt. Im Gegenteil, erfolgreichere Familienunternehmen setzen dieses intuitive Entscheidungsverhalten sogar signifikant häufiger ein als weniger erfolgreiche Familienunternehmen.

Während Familienunternehmen im Vergleich zu Nicht-Familienunternehmen (im Einklang mit Mintzberg und Waters 1982; Daily und Dollinger 1992) weniger auf formalisierte Steuerung setzen (kulturelle Steuerung und Ergebnissteuerung), setzen sie stärker auf Verhaltenskontrollen, was mit dem in früheren Arbeiten argumentierten Vorherrschen ,paternalistischer"/zentralisierter Entscheidungsstrukturen in Familienunternehmen (vgl. Dyer 1986) durchaus konsistent ist. Familienunternehmen delegieren unseren Daten zufolge weniger und zentralisieren Entscheidungen stattdessen stärker. Diese stärkere Zentralisierung von Entscheidungen geht einher mit einer geringeren Verwendung ergebnisbezogener Steuerungsinstrumente und expliziter „unternehmenskulturbezogener“ Steuerungsinstrumente. Unsere Daten bieten einen Ansatzpunkt, der auf ein Verbesserungspotential bei der Verhaltenssteuerung in Familienunternehmen hinweisen könnte: Tendenziell erfolgreichere Familienunternehmen setzen in größerem Umfang regelmäßig ergebnisorientierte Kennzahlen ein und setzen stärker auf eine explizite Formulierung und Kommunikation gemeinsamer Werte und Normen etwa in Form eines „Mission Statements“ oder eines „Code of Conduct“.

Die Ergebnisse unserer Studie untermauern, dass die geringere Nutzung von Entscheidungsunterstützungsinstrumenten auf Geschäftsführungsebene und die Besonderheiten der Verhaltenssteuerung in Familienunternehmen nicht pauschal auf mangelnde Professionalität im Management von Familienunternehmen zurückgeführt werden können. Besonderheiten von Familienunternehmen beim Einsatz von Entscheidungsunterstützungsund Verhaltenssteuerungsinstrumenten sind durch die Überlappung der Sphären Eigentum, Führung und Familie erklärbar, wobei die besondere Art der Führung von Familienunternehmen nicht nur Nachteile, sondern auch Vorteile hat (vgl. Habbershon und Williams 1999; Chrisman et al 2005; Sirmon und Hitt 2003). Ein intuitiveres Entscheidungsverhalten und ein direkteres und persönlicheres Vorgehen bei der Umsetzung von Unternehmenszielen und Strategien können insofern durchaus als Stärke von Familienunternehmen interpretiert werden (vgl. Mintzberg und Waters 1982). Die Besonderheiten der Entscheidungsfindung und Verhaltenssteuerung in Familienunternehmen sind dann nicht Ausdruck mangelnder Professionalität, sondern Ausdruck einer adäquaten Anpassung an die besonderen Rahmenbedingungen von Familienunternehmen.

Bei der Interpretation der empirischen Befunde der Untersuchung sind allerdings verschiedene Limitationen zu beachten. Der Fragebogen wurde nur von einer Person je Unternehmen (Geschäftsführer) bearbeitet, wodurch die Gefahr besteht, dass die Antworten die „subjektive Wahrnehmung des Respondenten“, nicht die „objektive Realität“ wider- 
spiegeln. Auf Grund der Tatsache, dass keine Längsschnittdaten vorliegen, sollten die empirischen Zusammenhänge nicht im Sinne von Ursache-Wirkungsbeziehungen interpretiert werden. Trotz der ergriffenen Maßnahmen zur Erhöhung der Reliabilität der Daten (randomisiertes Sample, Pre-Test des Fragebogens, Validierung der Konstrukte) kann die Messung der verwendeten Variablen/Konstrukte Verzerrungen unterliegen. Obwohl entsprechende Sensitivitätsanalysen ergaben, dass die Ergebnisse relativ stabil hinsichtlich alternativer Definitionen von Familienunternehmen sind, ist auch die spezielle gewählte Definition bei der Interpretation der Ergebnisse zu berücksichtigen.

Eine wesentliche Einschränkung stellt auch die Wahl des Maßes für die Unternehmensperformance dar. Da „objektive“ Performancemaße für kleine und mittlere Unternehmen bekanntermaßen schwer erhältlich und zudem wenig reliabel sind, wurde auf ein in der Literatur etabliertes Perzeptionsmaß zurückgegriffen (vgl. dazu Chenhall 2003).

In der bisherigen Literatur wurde zudem argumentiert, dass Instrumente der Entscheidungsfindung bzw. der Verhaltenssteuerung lediglich einen mittelbaren (etwa über die Entscheidungsqualität) Einfluss auf die Unternehmensperformance zeigen (Galinsky und Kray 2004; Widener 2007). Da auf Grund mangelnder Daten lediglich ein direkter Einfluss untersucht wurde, kann eine Verzerrung aufgrund nicht berücksichtigter Variablen nicht ausgeschlossen werden (Chenhall und Moers 2007). Schließlich ist zu berücksichtigen, dass die empirischen Daten von Unternehmen einer bestimmten Branche (Produktionsunternehmen) und aus einer bestimmten Region (deutschsprachiger Raum) stammen, was die Übertragbarkeit der Ergebnisse auf andere Branchen und Regionen limitiert.

\section{Anmerkungen}

1 Sowohl in Europa als auch in den USA können etwa 80-90 \% aller Unternehmen als Familienunternehmen klassifiziert werden und trotz der Dominanz kleinerer Familienunternehmen spielen diese auch als Arbeitgeber eine herausragende Rolle (vgl. etwa Astrachan und Shanker 2003; Burns und Whitehouse 1996; Chrisman et al. 2005).

2 In der Literatur zum Controlling (vgl. z. B. Ewert und Wagenhofer 2008; Weber und Schäffer 2008; Zimmerman 1997) werden die Entscheidungsunterstützung bzw. ,Rationalitätssicherung“ des Managements und die Steuerung des Verhaltens untergeordneter Manager und Mitarbeiter zur besseren Umsetzung der Unternehmensziele als die zwei Kernfunktionen von Controllingsystemen gesehen.

3 Die Beobachtungen sind voneinander unabhängig. Weiters folgen die verwendeten Konstrukte einer Normalverteilung, in den beiden untersuchten Gruppen (Familienunternehmen und NichtFamilienunternehmen) herrscht Homoskedastizität, d. h. die Varianzen der verwendeten Variablen sind in beiden Gruppen nicht signifikant unterschiedlich von einander.

4 Die Voraussetzung der Linearität wurde durch Analyse der Residuen überprüft, wobei keine nicht-linearen Muster zu erkennen waren. Dementsprechend kann von der Linearität der Regressionsgleichung ausgegangen werden (Hair et al. 2006). Ebenso erfolgte dieselbe Analyse für die einzelnen unabhängigen Variablen mithilfe von partiellen Regressionsplots, neuerlich ergaben sich keine nicht-linearen Muster. Ebenso ist Heteroskedastizität kein Problem in der Analyse, zumal die Residuen einigermaßen konstant sind für unterschiedliche Niveaus der unabhängigen Variablen, ebenso ergibt eine Analyse der Residuenplots eine Normalverteilung der Residuen. Um die Unabhängigkeit der Residuen zu testen, wurde ein Durbin-Watson Test berechnet, dessen Ergebnis mit 2,171 relativ nahe bei dem Wert zwei liegt und somit von einer Unabhängigkeit der Residuen ausgegangen werden kann (Field 2009). 


\section{Anhang}

Verwendete Konstrukte

\begin{tabular}{llll}
\hline Cronb. & Mean & Factor & Items \\
Alpha & (S.D.) & loading & \\
\hline
\end{tabular}

Verhaltensbeeinflussung durch Steuerung der Aufgabenverrichtung (action controls)

0,673 2,55 (1,62) 0,571 Auch bei geringfügigen Angelegenheiten muss die endgültige Entscheidung von einem Vorgesetzten genehmigt werden

3,12 (1,58) $\quad 0,865 \quad$ Unsere Mitarbeiter haben großen Freiraum bei der Gestaltung ihres Arbeitsalltags (umkodiert)

2,89 (1,36) $\quad 0,899 \quad$ Im Rahmen ihrer zugeordneten Aufgabengebiete treffen die Mitarbeiter ihre Entscheidungen vorwiegend selbst (umkodiert)

Verhaltensbeeinflussung durch Steuerung der Ergebnisse (results controls)

$0,875 \quad 5,13(1,54) \quad 0,884 \quad$ Kennzahlen (z. B. Umsatz, Produktqualität, etc.) werden verwendet zur laufenden Überwachung des Fortschritts der Zielerreichung

5,67 (1,25) 0,921 Kennzahlen (z. B. Umsatz, Produktqualität, etc.) werden verwendet zur Überprüfung der Ergebnisse

$5,55(1,29) \quad 0,891 \quad$ Kennzahlen (z. B. Umsatz, Produktqualität, etc.) werden verwendet zum Abgleich des Soll/Ist Zustands

Verhaltensbeeinflussung durch explizite kulturelle Steuerung (cultural controls)

0,830 5,37 (1,41) 0,911 Die Unternehmensziele und die zugrundeliegende Unternehmensphilosophie (Mission Statement) werden bewusst an die Mitarbeiter kommuniziert

$5,04(1,49) \quad 0,935 \quad$ In unserem Unternehmen wird immer wieder versucht, die Unternehmensphilosophie (Mission Statement) in das Gedächtnis der Mitarbeiter zu rufen

4,92 (1,36) 0,881 Unsere Unternehmensphilosophie (Mission Statement) ist unseren Mitarbeitern bewusst

3,61 (2,04) $\quad 0,618$ In unserem Unternehmen werden die Unternehmenswerte und informellen Verhaltensregeln (z. B. übliche Kommunikationsweise) in einem Verhaltenskodex (Code of Conduct) definiert

Rationalitätssicherung/Entscheidungsunterstützung in der Geschäftsführung (Selbstkontrolle)

$0,707 \quad 4,34(1,64) \quad 0,863 \quad$ Alle wichtigen Entscheidungen werden genau dokumentiert, sodass auch im Nachhinein genau nachvollziehbar ist, aufgrund welcher Daten und Kennzahlen die Entscheidung so getroffen wurde

$5,03(1,54) \quad 0,858 \quad$ Vor jeder wichtigen Entscheidung werden alle verfügbaren Daten und Kennzahlen durch die Geschäftsführung ausführlich analysiert und diskutiert

3,58 (1,64) $\quad 0,657$ Entscheidungen auf Geschäftsführungsebene werden häufig intuitiv aufgrund langjähriger Erfahrungswerte oder anhand zahlenmäßig nicht ausdrückbarer Einschätzungen der Geschäftsführung getroffen (umkodiert) 
(Fortsetzung)

\begin{tabular}{llll}
\hline Cronb. & Mean & Factor & Items \\
Alpha & (S.D.) & loading & \\
\hline
\end{tabular}

Umfeldunsicherheit

0,811 4,00 (1,44) 0,782 Das Kaufverhalten der Kunden unseres Unternehmens ist sehr gut abschätzbar (umkodiert)

3,38 (1,35) $\quad 0,844$ Technologische Veränderungen in der Hauptbranche unseres Unternehmens sind sehr gut abschätzbar (umkodiert)

$3,39(1,23) \quad 0,791 \quad$ Das Verhalten und/oder die Strategien der Lieferanten unseres Unternehmens sind sehr gut abschätzbar (umkodiert)

3,89 (1,29) $\quad 0,781 \quad$ Das Verhalten und/oder die Strategien der Konkurrenten unseres Unternehmens sind sehr gut abschätzbar (umkodiert)

\section{Exploration}

$0,754 \quad 4,23(1,75) \quad 0,795$

Wir haben uns Fähigkeiten zur Produktentwicklung (z. B. Produktdesign, Prototyping, Produktanpassungen an regionale Marktbedingungen) angeeignet, die in unserer Branche absolut neu sind

4,74 (1,41) $\quad 0,800 \quad$ Wir haben unsere Innovationsfähigkeit in Bereichen stark verbessert, in denen wir bisher keine Erfahrung hatten

3,97 (1,71) $\quad 0,873$ Wir haben komplett neue Fähigkeiten entwickelt, die wichtig für Innovationen sind (z. B. Vorhersage von technologischen Entwicklungen und Änderungen im Konsumentenverhalten, Management des Produktentwicklungsprozesses)

4,41 $(1,86) \quad 0,580 \quad$ Wir haben Fertigungstechnologien umgesetzt, die für unser Unternehmen völlig neu waren

\section{Exploitation}

$0,615 \quad 4,23(1,75) \quad 0,713$

Unser Unternehmen hat sein Wissen und seine Fähigkeiten zur Weiterentwicklung bestehender Produkte und Technologien kontinuierlich ausgebaut

4,74 (1,41) 0,704 Wir haben große Anstrengungen unternommen, um die Produktivität unserer Prozess- und Produktinnovationen zu verbessern

4,41 $(1,86) \quad 0,801 \quad$ Wir haben große Anstrengungen unternommen, um unsere bestehenden Forschungs- und Entwicklungsprozesse effizienter zu gestalten

\section{Unternehmenserfolg}

$0,891 \quad 5,31(1,27)$

0,924 Die allgemeine Entwicklung unseres Unternehmens im Vergleich zu unseren stärksten Wettbewerbern war sehr gut

$5,17(1,27) \quad 0,886 \quad$ Die finanzielle Entwicklung (Profitabilität) unseres Unternehmens im Vergleich zu unseren stärksten Wettbewerbern war sehr gut

$5,29(1,26) \quad 0,909 \quad$ Die Umsatzentwicklung unseres Unternehmens im Vergleich zu unseren stärksten Wettbewerbern war sehr gut 


\section{Literatur}

Armstrong JS, Overton TS (1977) Estimating nonresponse bias in mail surveys. J Mark Res 14: 396-402

Astrachan JH, Shanker MC (2003) Family businesses' contribution to the U.S. Economy: a closer look. Fam Bus Rev 16:211-219

Backhaus K, Erichson B, Plinke W, Weiber R (2003) Multivariate Analysemethoden, 10. Aufl. Springer, Berlin

Bracker JS, Keats BW, Pearson JN (1988) Planning and financial performance among small firms in a growth industry. Strat Manag J 9:591-603

Burkart M, Panunzi F, Shleifer A (2003) Family firms. J Financ 58(5):2167-2202

Burns P, Whitehouse O (1996) Family ties. Special Report of the 3rd European Enterprise Center. Milton Keynes: 3rd European Enterprise Center

Carrasco-Hernandez A, Sanchez-Marin G (2007) The determinants of employee compensation in family firms: empirical evidence. Fam Bus Rev 20(3):215-228

Chandler AD (1977) The visible hand: the managerial revolution in American business. Harvard University Press, Cambridge

Chandler AD (1990) Scale and scope: the dynamics of industrial capitalism. Harvard University Press, Cambridge

Chenhall RH (2003) Management control systems design within its organizational context: findings from contingency-based research and directions for the future. Acc Organ Soc 28(2-3):127-168

Chenhall RH, Moers F (2007) The issue of endogeneity within theory-based, quantitative management accounting research. Eur Acc Rev 16(1):173-196

Chrisman JJ, Chua JH, Sharma P (2005) Trends and directions in the development of a strategic management theory of the family firm. Entrep Theory Pract 29(5):555-575

Chua JH, Chrisman JJ, Sharma P (1999) Defining the family business by behavior. Entrep Theory Pract 23:19-39

Daily CM, Dollinger MJ (1992) An empirical examination of ownership structure in family and professionally-managed firms. Fam Bus Rev 5(2):117-136

Davila A (2005) An exploratory study on the emergence of management control systems: formalizing human resources in small growing firms. Acc Organ Soc 30(3):223-248

Davila A, Foster G (2007) Management accounting systems' adoption decisions: evidence and performance implications from startup companies. Acc Rev 80(4):1039-1068

Donaldson L (2001) The contingency theory of organizations. Sage, California

Dyer WG (1986) Cultural change in family firms: anticipating and managing business and family transactions. Jossey-Bass, San Francisco

Ewert R, Wagenhofer A (2008) Interne Unternehmensrechnung, 7. Aufl. Springer, Berlin

Fama E, Jensen M (1983) Separation of ownership and control. J Law Econ 26:301-326

Field A (2009) Discovering statistics using SPSS, 3. Aufl. Sage

Flamholtz EG (1986) Managing the transition from an entrepreneurship to a professionally managed firm. Jossey-Bass, San Francisco

Galinsky AD, Kray LJ (2004) From thinking about what might have been to sharing what we know: the effects of counterfactual mind-sets on information sharing in groups. J Exp Soc Psych 40(5):606-618

Garson DG (2008) http://faculty.chass.ncsu.edu/garson/PA765/manova.htm. Zugegriffen: 28. Okt. 2010

Geeraerts G (1984) The effect of ownership on the organization structure in small firms. Adm Sci Q 29(2):232-237

Habbershon TG, Williams ML (1999) A resource based framework for assessing the strategic advantages of family firms. Fam Bus Rev 12(1):1-25 
Hair J, Black WC, Babin BJ, Anderson RE, Tatham RL (2006) Multivariate data analysis, 6. Aufl. Prentice Hall, Upper Saddle River

Hall A, Nordqvist M (2008) Professional management in family businesses: toward an extended understanding. Fam Bus Rev 21(1):51-69

Harris RID, Reid RS, McAdam R (2004) Employee involvement in family and non-family-owned businesses in Great Britain. Int J Entrep Behav Res 10(1/2):49-58

Hartmann FGH, Moers F (1999) Testing contingency hypotheses in budgetary research: an evaluation of the use of moderated regression analysis. Acc Organ Soc 24(4):291-315

Henri JF (2006) Management control systems and strategy: a resource-based perspective. Acc Organ Soc 31:529-558

Jorissen A, Laveren E, Martens R, Reheul AM (2005) Real versus sample-based differences in comparative family business research. Fam Bus Rev 18(3):229-246.

Kets de Vries MFR (1993) The dynamics of family controlled firms: the good and the bad news. Organ Dyn 21(3):59-71

Kotey B (2005) Are performance differences between family and non-family SMEs uniform across all firm sizes? Int J Entrep Behav Res 11(6):394-421

McEachern WA (1978) Corporate control and growth: an alternative approach. J Ind Econ 26(3): $257-266$

Menard S (1995) Applied logistic regression analysis. Sage University Series, Thousand Oaks

Merchant K, Van Der Stede WA (2007) Management control systems, 2. Aufl. Prentice Hall, Harlow

Minichilli AG, Corbetta G, MacMillan IC (2010) Top management teams in family-controlled companies: 'Familiness', 'Faultlines', and their impact on financial performance. J Manag Stud 47(2):205-222

Mintzberg H, Waters J (1982) Tracking strategy in an entrepreneurial firm. Acad Manag Rev 25(3):465-499

Moers F (2006) Performance measure properties and delegation. Acc Rev 8(4):897-924

Moores K, Mula J (2000) The salience of market, bureaucratic, and clan controls in the management of family firm transitions: some tentative australian evidence. Fam Bus Rev 13(2):91-106

Moores K, Yuen S (2001) Management accounting systems and organizational configuration: a life-cycle perspective. Acc Organ Soc 26(4-5):351-389

Ouchi WG (1979) A conceptual framework for the design of organizational control mechanisms. Manag Sci 25(9):833-848

Schulze WS, Lubatkin MH, Dino RN, Buchholtz AK (2001) Agency relationships in family firms: theory and evidence. Organ Sci 12(2):99-116

Sharma P (2004) An overview of the field of family business studies: current status and directions for the future. Fam Bus Rev 17(1):1-36

Sirmon D, Hitt MD (2003) Managing resources: linking unique resources, management and wealth creation in family firms. Entrep Theory Pract 27(4):339-365

Speckbacher G, Wentges P (2011) The impact of family control on the use of performance measures in strategic target setting and incentive compensation: a research note. Manag Acc Res 23(1): 34-36

Tagiuri R, Davis J (1996) Bivalent attributes of the family firm. Fam Bus Rev 9(2):199-208

Weber J, Schäffer U (2008) Einführung in das Controlling, 12. Aufl. Schäffer Poeschl, Stuttgart

Widener S (2007) An empirical analysis of the levers of control framework. Acc Organ Soc 32: 757-788

Zahra SA, Hayton JC, Salvato C (2004) Entrepreneurship in family vs. non-family firms: a resourcebased analysis of the effect of organizational culture. Entrep Theory Pract 28(4):363-381

Zahra SA, Ireland RD, Hitt MD (2000) International expansion by new venture firms. Acad Manag J 43(5):925-950

Zimmerman JL (1997) Accounting for decision-making and control. McGraw-Hill, Chicago 


\title{
Management control in family firms: idiosyncrasies of decision-facilitating and decision-influencing roles and their effect on performance
}

\begin{abstract}
This paper draws on a survey design to investigate whether family ownership and leadership have an influence on the decision-facilitating and decision-influencing roles of management control. Moreover, we investigate whether the more intuitive decision making in family firms which is often said to be caused by a lack of professionalism, is indeed a disadvantage. The results indicate significant differences in the use of management controls between family firms and non-family firms. However, in contrast to the prevailing view in literature, the performance of family firms is not affected negatively by their specific way of making decisions and by their more centralized management style. According to our data, the prevailing characterization of family firms as being less professionally managed seems questionable.
\end{abstract}

Keywords: Management control $\cdot$ Family firms $\cdot$ Decision-facilitating role $\cdot$ Decision-influencing role 